Cahiers
Recherche
surles Droits Fondamentaux

Cahiers de la recherche sur les droits fondamentaux

4 | 2005

Quel avenir pour la laïcité cent ans après la loi de 1905 ?

\title{
Le Code civil et le juge administratif
}

Xavier Mondésert

\section{(2) OpenEdition}

Journals

Édition électronique

URL : https://journals.openedition.org/crdf/7378

DOI : $10.4000 /$ crdf. 7378

ISSN : 2264-1246

Éditeur

Presses universitaires de Caen

Édition imprimée

Date de publication : 1 décembre 2005

Pagination : 179-182

ISBN : 2-84133-250-0

ISSN : $1634-8842$

Référence électronique

Xavier Mondésert, « Le Code civil et le juge administratif », Cahiers de la recherche sur les droits

fondamentaux [En ligne], 4 | 2005, mis en ligne le 15 décembre 2020, consulté le 14 novembre 2022

URL : http://journals.openedition.org/crdf/7378; DOI : https://doi.org/10.4000/crdf.7378 


\title{
Le Code civil et le juge administratif
}

\author{
Xavier MONDÉSERT
}

\author{
I. Le Code civil inspire le juge administratif \\ II. Le juge administratif applique le Code civil
}

Au premier abord, il peut paraître surprenant qu'un publiciste prenne la parole à l'occasion du bicentenaire du Code civil. Mais, à la réflexion, si l'on veut apprécier l'importance et la portée réelle de ce code dans la vie juridique, son rayonnement, il faut nécessairement déborder la jurisprudence de la Cour de cassation; car il n'y a rien d'étonnant à ce que celle-ci en fasse application. En revanche, il n'est pas inutile de se demander comment le juge administratif prend en compte le Code civil. D'ailleurs, il eût été également intéressant de s'interroger sur la place du code dans la jurisprudence constitutionnelle.

Mon propos sera limité par ma compétence et consacré aux différentes démarches qui sont empruntées par le Conseil d'État lorsqu'il rencontre le Code civil au contentieux. Mais n'oublions pas le rôle qu'a également joué le Conseil dans ses formations consultatives, lors de l'élaboration du Code civil et de ses révisions postérieures.

Le premier des grands arrêts de la jurisprudence administrative, la fameuse décision Blanco rendue par le Tribunal des conflits le 8 février 1873 (Lebon ${ }^{\text {er }}$ supplément p. 61), écarte l'application du Code civil par une rédaction de principe particulièrement claire : «Considérant que la responsabilité, qui peut incomber à l'État pour les dommages causés aux particuliers par le fait des personnes qu'il emploie dans le service public, ne peut être régie par les principes qui sont établis dans le Code civil, pour les rapports de particuliers à particuliers... ». Il ne faudrait pas extrapoler cette solution limitée à la responsabilité de la puissance publique; il ne faudrait pas en déduire que le Conseil d'État croit pouvoir ignorer de manière générale et absolue le Code civil. En réalité, le juge administratif cherche simplement à vérifier l'adéquation des règles du droit privé aux situations de droit public pour pouvoir déterminer s'il convient de les appliquer ou de les écarter ; cette démarche n'implique ou n'entraîne aucune méfiance de sa part envers le Code civil. D'ailleurs, bien souvent, les jurisprudences judiciaire et administrative se rejoignent, on l'oublie à trop vouloir souligner les divergences; par exemple, l'arrêt de la Cour de cassation du 23 novembre 1956, D ${ }^{r}$ Giry (Grands arrêts de la jurisprudence administrative, $\mathrm{n}^{\circ} 77$ ), reprend la solution de l'arrêt Blanco : «Attendu que la Cour d'appel s'est appuyée, à tort, sur les dispositions de droit privé relatives aux délits et quasidélits, qui ne peuvent être invoqués pour fonder la responsabilité de l'État; qu'elle avait, en revanche, le pouvoir et le devoir de se référer, en l'espèce, aux règles du droit public».

Le Conseil d'État, pour utiliser le Code civil, met en œuvre six méthodes différentes qui lui laissent une plus ou moins grande marge de manouvre. Nous allons constater que, grâce à ces méthodes, le Code civil est souvent une source d'inspiration pour le juge administratif, d'une part, et que celui-ci peut parfois être amené à faire application des règles du droit civil, d'autre part.

\section{Le Code civil inspire le juge administratif}

Cette influence est double ; elle est d'ordre général et presque philosophique en premier lieu, d'ordre plus juridique et technique en second lieu.

Une inspiration d'ordre plutôt philosophique d'abord, en ce que le Conseil d'État va rechercher dans le Code civil 
un point de vue général, non purement abstrait, mais en référence à des règles juridiques. Le Code, dans cette première perspective, présente pour le Conseil d'État le caractère d'un texte révélateur de valeurs tellement essentielles qu'elles se trouvent enracinées dans le droit, surtout en des domaines qui relèvent de l'état des personnes. C'est ainsi qu'à l'occasion d'une affaire relative à la sanction disciplinaire prononcée à l'égard d'un médecin qui avait pratiqué une expérimentation sur un patient en état de mort cérébrale, alors que l'arrêt lui-même n'est fondé sur aucune disposition du Code civil qui était à l'époque juridiquement inapplicable, le commissaire du gouvernement a proposé au Conseil d'État de se tourner vers ce code pour tenter de cerner la notion de cadavre: «l'approche civiliste permet en effet de mieux mesurer ce qu'est au regard de notre droit le corps humain avant et après la mort » (CE Ass. 2 juillet 1993, Milhaud, Lebon p. 194, concl. Kessler p. 201). Et, au requérant qui soutenait que l'inviolabilité du corps humain ne serait que l'expression d'un préjugé, le commissaire du gouvernement a pu ainsi répondre qu'au contraire cette indisponibilité découle aussi bien de valeurs culturelles que des principes de notre droit positif qui n'assimilent pas le corps à un objet (ibid., p. 203). Dans cette mesure, la décision du Conseil d'État, motivée par les principes déontologiques fondamentaux qui s'imposent au médecin, pour prétorienne qu'elle soit, n'est pas arbitraire. La jurisprudence administrative est pour l'essentiel conforme à l'esprit de la loi civile puisque cet arrêt est antérieur à la loi du 29 juillet 1994 relative au respect du corps humain, qui a introduit dans le Code civil un article 16 interdisant toute atteinte à la dignité de la personne; depuis, cet article $16 \mathrm{du}$ Code civil est directement appliqué par le Conseil d'État (par exemple, dans son récent arrêt du 29 septembre 2004, Union française pour la cohésion nationale).

Cette première démarche du Conseil d'État n'est pas récente: dès 1909, à une époque où la Doctrine redécouvrait l'arrêt Blanco trente ans après pour fonder le modèle classique du service public alors triomphant, un commissaire de gouvernement concluant sur l'arrêt Winkell portant en matière de grève des fonctionnaires, matière pourtant administrative par nature, n'hésitait pas à soutenir qu' «il importe au Conseil d'État de connaître et d'étudier de près la jurisprudence de la Cour de cassation concernant les effets juridiques de la grève sur le contrat de travail » (CE 7 août 1909, Winkell, Lebon p. 1293, concl. Tardieu p. 1300).

Une inspiration d'ordre plus juridique ensuite, en ce que le Conseil d'État, d'une part, peut étendre à des situations de droit public, de manière mesurée, certains principes formulés dans le Code civil ; mais ces principes ne sont pas appliqués en tant que tels car les dispositions du code, selon cette deuxième démarche du Conseil, ne feraient que s'inspirer de principes de portée plus générale relevant également du droit public. C'est ainsi que, dans la jurisprudence administrative, le régime de la garantie décennale des architectes et des entrepreneurs découle des " principes dont s'inspirent les articles 1792 et $2270 \mathrm{du}$ Code civil» (CE Ass. 2 février 1973, Trannoy, Lebon p. 95).
C'est encore ainsi que, dans un arrêt de section du 28 juin 1996, M. Krief, le Conseil d'État énonce « qu'il résulte du principe dont s'inspire l'article 1326 du Code civil que toute convention comportant un engagement unilatéral de payer une somme d'argent doit, dans l'intérêt de la personne qui le souscrit, exprimer de façon non équivoque la connaissance que celle-ci a de la nature et de l'étendue de son obligation » (JCP, 1996-II-22704, p. 385). Cette technique particulière permet au juge administratif de transposer un régime des principes propres au Code civil, sans se lier par la lettre de ce code ni par les interprétations qu'en donne le juge judiciaire.

D'autre part, le Conseil d'État par sa troisième méthode va parfois faire application de techniques et de notions qui dépassent la division droit privé / droit public, même si elles se trouvent formulées dans le Code civil. C'est pourquoi, selon le commissaire du gouvernement Combrexelle dans ses remarquables conclusions sur la décision précitée de 1996, $M$. Krief, les arrêts généralement ne font pas expressément référence au code, signifiant par là que ces techniques et notions trouvent leur origine dans un fonds commun le plus souvent inspiré du droit romain, qui est indistinctement utilisé par le juge judiciaire, par le juge administratif mais aussi par les conventions internationales. Si l'on s'en tient (comme M. Combrexelle) au seul droit des obligations, force est de constater que la jurisprudence est à la fois ancienne et abondante: on peut citer, à titre d'exemple de ces techniques et notions communes au droit privé et au droit public [en se référant seulement à la date de l'arrêt inaugural, pour bien marquer l'évolution chronologique], l'erreur sur la substance ou l'objet du contrat (CE 24 juillet 1885, Ville de Vichy, Lebon p. 723), la force obligatoire du contrat (CE 17 mars 1893, $C^{i e} d u$ Nord, S, 1894, 3, p. 119), la nullité des contrats fondés sur une cause illicite (CE 25 novembre 1921, $S^{t e}$ des savonneries Henri Olive, Lebon p. 977), le dol (CE 14 décembre 1923, $S^{t e ́}$ des Grands Moulins de Corbeil, Lebon p. 852), l'action oblique (CE Sect. 18 mars 1927, Fauvet, Lebon p. 360), la transaction (CE Sect. 9 février 1934, Chemins de fer $d u$ Nord c. Sieur de Brigode, Lebon p. 194), la compensation (CE 4 décembre 1935, Bernard, Lebon p. 1130 : « les règles spéciales de la comptabilité publique ne font pas obstacle à la compensation de la dette susvisée du Trésor avec sa créance liquide et exigible »), la violence comme vice du consentement (CE 19 janvier 1945, $S^{t e ́}$ des aéroplanes Voisin, Lebon p. 19), l'action paulienne (CE Sect. 21 juin 1957, Dupuy, Lebon p. 411), l'enrichissement sans cause (CE Sect. 14 avril 1961, Ministre de la reconstruction et du logement c. S $S^{t e ́}$ Sud-aviation, Lebon p. 236, l'arrêt précisant qu'il s'agit d'un «principe applicable, même sans texte, à la matière de travaux publics »), la force majeure (CE 24 avril 1968, $C^{i e}$ de navigation Denis Frères, Lebon p. 257), la subrogation (CE Sect. 13 octobre 1972, Caisse régionale de réassurances mutuelles agricoles de l'Est, Lebon p. 635), l'effet relatif des contrats (CE Sect. 29 juin 1973, Ministre de l'équipement et du logement c. $S^{\text {té }}$ parisienne pour l'industrie électrique, Lebon p. 457), la solidarité (CE Sect. 9 janvier 1976, $S^{\text {té }}$ Caillol et autres, Lebon p. 19), la liberté contractuelle (CE Sect. 28 janvier 1998, Société Borg Warner, 
Lebon p. 20, cet arrêt faisant simultanément application de la liberté contractuelle, sans référence à un texte, et de la garantie décennale dont s'inspirent les articles 1792 et suivants, ce qui prouve bien que les deux méthodes sont différentes). Cette démarche du Conseil d'État est donc fréquente et constante.

Mais le Code civil non seulement inspire le Conseil d'État, mais il participe également, dans une certaine mesure, à l'élaboration de la jurisprudence administrative.

\section{Le juge administratif applique le Code civil}

En premier lieu, le Conseil d'État en fait une libre application lorsqu'il met en œuvre une véritable démarche de politique jurisprudentielle; en second lieu, il en fait une application plus contrainte quand il s'estime lié par la lettre du Code.

D'abord, dans le cadre d'une démarche de politique jurisprudentielle, quatrième méthode qui relève encore d'une appréciation assez libre, le Conseil d'État peut faire, à des rapports de droit public, une application directe et littérale de certaines règles du Code civil et ce, sans justification explicite, en usant de son pouvoir prétorien. On peut citer à titre d'exemple l'article 1153 sur les intérêts moratoires (voyez l'arrêt de principe récent, d'autant plus remarquable qu'il a été rendu en matière fiscale, réputée particulièrement « autonome »: CE 30 juin 2004, $S^{t e ́} A k s o$ Nobel, Rev. de Dr. fisc., 2004 / 41758), l'article 1154 sur la capitalisation des intérêts (CE 4 novembre 1959, Commune de Bollène, Lebon p. 575), les articles 1244, 1253 et suivants sur l'imputation des versements faits par un débiteur à son créancier en cas de pluralité de dettes (CE 29 juin 1964, X, Lebon p. 367), l'article 1601-3 sur le contrat de vente en l'état futur d'achèvement (CE Sect. 8 février 1991, Région Midi-Pyrénées, Lebon p. 41), l'article 2061 sur l'interdiction de la clause compromissoire (CE Sect. 3 mars 1989, $S^{\text {té }}$ des autoroutes de la région Rhône-Alpes, Lebon p. 69), l'article 2244 sur l'interruption du délai de prescription par une action en référé (CE avis 22 juillet 1992, Commune de Marcilly-sur-Eure, Lebon p. 305), l'article 2262 sur la prescription trentenaire en matière de recouvrement des créances ni fiscales ni domaniales (CE Ass. 13 mai 1960, Secrétaire d'État à l'agriculture c. Manière, Lebon p. 328). L'application littérale des dispositions du Code civil n'est pas réservée au contentieux contractuel, le juge de l'excès de pouvoir exerce également son contrôle de la légalité des actes administratifs au regard des lois à caractère principalement civil (Raymond Odent, Contentieux administratif, $2^{\mathrm{e}}$ éd., Paris, Les Cours de Droit, fascicule V, p. 1678).

Le problème est bien évidemment de déterminer pourquoi certaines dispositions du Code civil sont écartées par le juge administratif et pourquoi d'autres sont interprétées comme énonçant un principe général ou sont appliquées en tant que telles. Comme l'enseigne le Président Odent (fascicule I, p. 14), «chaque fois qu'aucune considération tirée de la structure ou des conditions de fonctionnement des services publics n'y fait obstacle, ce ne sont pas seulement les principes du droit civil, ce sont ses textes mêmes qui s'appliquent en droit public »; et le commissaire du gouvernement Combrexelle ajoute que le juge administratif incorpore dans sa propre jurisprudence une disposition du Code civil «si elle est à la fois utile en ce qu'elle permet de pallier un vide juridique, compatible avec les exigences du service public et cohérente avec les autres règles et principes du droit administratif» (conclusions précitées, p. 386).

Cette politique jurisprudentielle n'est d'ailleurs pas réservée par le Conseil d’État au droit civil puisqu'elle a joué, mais plus tardivement qu'à l'endroit du Code civil, également à l'égard du droit pénal (CE Ass. 6 décembre 1996, $S^{t e ́} L a m b d a$, Lebon p. 466), du droit de la concur-

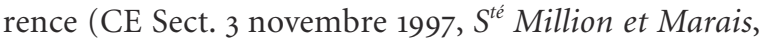
Lebon p. 406), du droit des assurances (CE 29 décembre 2000, Beule et autres, Lebon p. 656) ou encore à l'égard du droit de la consommation (CE Sect. 11 juillet 2001, $S^{t e ́}$ des eaux du Nord, Lebon p. 348, le commissaire du gouvernement indiquant: " nous ne voyons pas d'obstacle à ce que vous fassiez une application directe du code de la consommation, sans qu'il soit nécessaire de se borner à se référer aux principes qu'il dégage », p. 356). Le bloc de légalité formant un tout, le juge administratif est soucieux de sa cohérence (notons néanmoins que le Conseil d'État écarte l'application du Code du travail qui contient des dispositions radicalement inapplicables aux agents publics : CE Ass. 8 juin 1973, Dame Peynet, Lebon p. 406, sauf exceptions : art. L. 521-2 et suivants relatifs à la grève dans certains services publics).

Enfin, la simple logique juridique impose parfois au Conseil d'État d'appliquer littéralement les dispositions de droit public qui sont incluses dans le Code civil ; ce sera sa cinquième méthode. En effet, certaines règles du code visent des situations qui ne relèvent pas du droit privé: par exemple, l'article 2060 qui interdit aux établissements publics de compromettre. Faut-il s'en étonner? Le professeur Demolombe a publié en 1845 un Cours de Code civil, dans le premier tome duquel il observe qu' « il serait impossible d'énumérer ici toutes les dispositions qui appartiennent au droit public... Il s'en rencontre dans presque tous les codes, un peu plus ou un peu moins; et quelquefois même des distinctions peuvent être nécessaires pour faire, dans le même sujet, la part du droit public et celle du droit privé» (Paris, Auguste Durand, p. 18). Sans dresser la liste exhaustive des dispositions du Code qui relèvent du droit public, et pour se borner donc à illustrer la jurisprudence du Conseil d'État, on citera le domaine public mentionné notamment aux articles 538 à 542 du Code civil (CE 31 mai 1989, Mazaurin, Lebon p. 137) ou l'interdiction faite par l'article 1596 aux administrateurs des biens communaux de se rendre acquéreurs des biens confiés à leur garde (CE 25 mars 1987, Delerue, Lebon p. 106).

En revanche, une sixième et dernière méthode consiste en l'application pure et simple de règles précises du Code civil qui, quant à elles, visent des situations régies, non plus par le droit public, mais exclusivement par le droit civil, situations que le droit administratif doit parfois obligatoirement prendre en compte. Par exemple, l'ancien 
article 216 du Code civil, qui permettait au mari de s'opposer à ce que son épouse exerce une profession séparée, s'appliquait aux fonctions publiques comme aux professions privées (CE 19 février 1943, Pagès, Lebon p. 44). Le Conseil d'État a plus récemment annulé un décret réservant un avantage aux fonctionnaires de sexe masculin au titre de leurs épouses et de leurs enfants, au motif que la loi du 4 juin 1970 a substitué aux dispositions de l'article 213 du Code civil, qui énonçaient que « le mari est le chef de famille», des dispositions nouvelles aux termes desquelles « les époux assurent ensemble la direction morale et matérielle de la famille » (CE 28 juillet 1993, $M^{m e}$ Dupuy, Lebon p. 244). De la même manière, le Conseil d'État a annulé le règlement intérieur exigeant dans un lycée l'autorisation des parents pour des élèves âgés de plus de dixhuit ans, au motif que cet acte méconnaissait les dispositions de l'article 488 du code relatif à la majorité (CE Sect. 22 mars 1996, $M^{\text {me }}$ Paris et $M^{\text {me }}$ Roignot, Lebon p. 99).

Ces différentes démarches, qui concernent ainsi de nombreuses dispositions du Code civil, sont mises en œuvre de manière pragmatique par le Conseil d'État selon les circonstances de chaque affaire à juger. On peut donner une idée de la fréquence d'utilisation du Code civil, par application de l'une de ces démarches, en indiquant qu'une analyse rapide des arrêts figurant dans les banques de données juridiques montre que, chaque année, sur les quelques 5000 décisions du Conseil d'État, de 50 à 100 arrêts environ se fondent, dans leurs motifs, sur une disposition du Code civil, soit un taux de l'ordre de 1 à $2 \%$. Ce qui signifie que, bien entendu, le principe de la liaison de la compétence et du fond demeure encore valable; pour l'essentiel, c'est bien au juge judiciaire qu'il incombe d'appliquer le Code civil. Mais on ne peut pas dire que le Conseil d'État ignore superbement ce code.

L'unification des règles de droit civil au sein du Code civil n'a donc pas porté atteinte à l'unité du droit dans son ensemble, par-delà même la distinction académique entre droit public et droit privé. L'inscription du droit civil dans le marbre d'un code n'a pas pétrifié la jurisprudence. Et c'est un constat rassurant car, quel que soit le juge appelé à trancher un litige, le droit appliqué ne doit pas être un droit fragmenté; la culture juridique ne peut pas se réduire à des connaissances fragmentaires.

«D'ailleurs », écrit Borgès (le maître de la littérature argentine) dans une de ses nouvelles, «ce qui importe ce n'est pas de lire mais de relire» ( UUtopie d'un homme qui est fatigué », in Le Livre de sable, Paris, Gallimard, 1983, p. 105). Nous avons tous lu le Code civil, peu ou prou; nous devons le relire. Relisons-le encore, relisons-le toujours! 